New Media

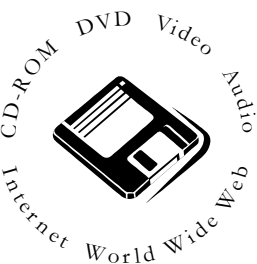

\section{CD-ROM Review}

(1) Arterial Blood Gas Interpretation (SimBiosys ABG) $V: 2$ (1997)

"An Interactive Tutorial for Blood Gas and Acid-Base Analysis"

Critical Concepts Inc., Chicago, USA

www.critcon.com

ISBN 10890404-06-3

$\$ 74.00$ USD

This Windows program teaches the key concepts of arterial blood gas (ABG) interpretation to a level suitable for studying for the FRCPC examination (in virtually all disciplines). I had no trouble whatsoever installing the program and learning to use it. After installation, the user is offered an opening menu, which either allows one to read material on ABG physiology and pathophysiology or allows one to interact with a variety of clinical cases ( 48 in all). Since the text part is just as boring on a video screen as printed on paper, I soon found myself playing with various clinical scenarios by answering questions as to what I thought was going on metabolically in cases such as heroin or aspirin overdoses. I quickly found that my performance on some of these cases was somewhat less than stellar, although I admit I didn't try as hard as I might have. Still, I found that working through the cases was a great way to study the topic. My only complaint was that I was marked wrong on one question where I maintained that my patient was not hypoxemic with an arterial oxygen tension of $62 \mathrm{mmHg}$ (almost all textbooks use 60 as the threshold for hypoxemia).

The text sections are connected by hyperlinks, so that it is very easy to get more details quickly. The text material is also accompanied by real time (dynamically adjustable) diagrams covering concepts such as oxygen content of blood, the oxyhemoglobin dissociation curve or the calculation of venous admixture. (By dynamically adjustable I mean that the user can adjust model parameters such as hemoglobin concentration with a scroll-bar to see what happens. This is a great interactive feature that is not possible with ordinary teaching materials).
A particularly interesting aspect of this package is that it allows you to enter your own clinical data to obtain detailed clinical commentaries, including animated diagrams.

Topics covered include: shunt, venous admixture, ventilation-perfusion matching, the oxyhemoglobin dissociation curve, $\mathrm{CO}_{2}$ dissociation equilibrium and more. Acid-Base diagrams are available in six different forms and one can switch among them by clicking on tabs on the right side of the panel.

In conclusion, SimBiosys ABG is a Windows program that offers an interactive and dynamic approach to $A B G$ interpretation, including cases upon which you can be quizzed. Extensive on-screen tutorials use hyperlinks and real-time simulation to offer an enjoyable learning experience to cover an otherwise very dry subject. Highly recommended.

\section{John Doyle MD}

Toronto, Ontario

\section{(2) Robots, Software Agents, Softbots and the Internet}

An agent is an individual who acts on your behalf, with your authority, such as a lawyer who assists you in negociating a business deal. For some years the agent concept has now been extended to the computer world, where a more comprehensive definition might simply be "an agent is any entity that acts on behalf of another". Sometimes software agents are known as "Softbots" or simply "Bots". In short, they are software tools for digging through data - you give a Bot directions and it brings back answers.

The idea of robots as human-like machines was first conceived in Karel Capek's 1921 play "R.U.R.," where the playwright introduced Rossum's Universal Robots. The robot concept was later made even more famous by writer Isaac Asimov, beginning with his story "I, Robot" (1950) and continuing through a string of books known as the Robot Series.

On the Web, robots have taken on a new form of life in a software-only embodiment. Since all Web 
servers are connected on the Internet, robot-like software is an ideal way to perform Internet searches for all kinds of information.

Most people who use the Internet extensively have at one time or another been exposed to Bots, given that software agents are simply computer systems to which one can delegate tasks, such as helping one find clinical materials to help manage a puzzling medical case or even finding a good price for a vacation package meeting your specifications. Bot types as listed at www.botspot.com include: Chat Bots, Commerce Bots, Data Mining Bots, E-Mail Bots, Fun Bots, Game Bots, Government Bots, Knowledge Bots, News Bots, Search Bots, Shopping Bots, Software Bots, Stock Bots, Surveillance Bots, and Update Bots. For example, Web search engines send out Search Bots that "crawl" from one server to another, compiling an enormous list of URLs and information about content that is central to every search engine. By contrast, a Game Bot is a computer-controlled game opponent, usually programmed to exhibit some level of artificial intelligence (AI) while playing against humans in multiplayer computer games. Shopping bots compile databases about products sold at online stores. As an example, PricingCentral.com, an online price comparison Shopping Bot, produces a comprehensive directory to help one find the best bargains when shopping on the Internet.

Technically speaking, software agents differ from conventional software "in that they are long-lived, semi-autonomous, proactive, and adaptive". They may be thought of as a computer program "whose execution is contingent upon events and data conditions in its environment and is not under continuous, direct control by a human user". A software agent necessarily has some degree of autonomy but must at least occasionally interact with its master to report on progress made or to request additional guidance. In situations where a number of agents may be working concurrently, there sometimes exists a need for them to communicate using an "agent communication language" such as the emerging standard known as KQML.

I expect that there are likely to be many kinds of useful robots available in the near future designed to assist us in countless clerical tasks. Most futurists never imagined that the really useful robots would have no physical embodiment and would not even be able to help us clean our house!

D. John Doyle MD

Toronto, Ontario
(3) The Postmodernism Generator Web Site http://www.elsewhere.org/cgi-bin/postmodern/

This web site uses Artificial Intelligence (AI) software techniques to generate completely meaningless essays on a random basis, all the while adhering to a syntactically-perfect rigorous postmodern academic style. (Postmodernism emphasises the cultural relativity of all knowledge, including scientific knowledge, and holds in particular that scientific knowledge is not objective.) For the many scientists and philosophers who hold that postmodern philosophy is intellectually bankrupt, this parody site is an absolute delight. The generated essays are similar to critically-acclaimed "abstract art" randomly produced by a team of monkeys given paint brushes and a set of paints.

\section{John Doyle MD}

Toronto, Ontario 